\title{
The Research on the Singing Voice Timbre of the Eastern Yugur Traditional Folk Songs
}

\author{
Shiliang Lyu ${ }^{1, a}$ \\ ${ }^{1}$ Key Laboratory of National Language Intelligent Processing, Gansu Province, Northwest MinZu \\ University, Lanzhou, China \\ ashilianglv@126.com
}

Keywords: Yugur traditional folk songs; Singer's formant; Pitch frequency; Acoustic analysis

\begin{abstract}
The Yugur folk songs with distinctive ethnic characteristics. Traditional folk songs retain more features of minority folk songs, and have important research value and cultural value. The eastern folk songs due to historical and geographical factors, and in the process of language integration, with the influence of Mongolian folk songs, the folk songs produced unique singing characteristics. This paper uses the research the method of speech acoustics, research on Eastern Yugur traditional folk songs singing voice timbre, including two aspects of singer's formant and singing voice frequency characteristics. The study concluded that the folk songs singing timbre features and singer singing characteristics.
\end{abstract}

\section{Introduction}

The traditional folk songs with the unique artistic style and aesthetic characteristics. Yugur is a minority of our country, which is restricted by history, economy and geography, and the language of Yugur is divided into two kinds: eastern and western. Yugur folk songs due to language differences, also divided into eastern folk songs and western folk songs. Eastern Yugur belongs to the Altaic language family of Mongolian, western Yugur belongs to the Altaic language Turkic family. Affected by the language and the living environment, many scholars think that the eastern part of the Yugur folk songs close to the Mongolian folk songs, and the western folk more to retain the characteristics of the Yugur nationality. Among them, in the process of language interaction and blending, the eastern Yugur's folk songs by Mongolian and folk songs have changed. Therefore, the eastern Yugur folk songs have important research value.

Timbre is the quality and personality of sound, it is one of the four elements of speech. In the four elements of speech, pitch reflects the level of the sound, depending on the frequency of the vibration of the vocal cords. The sound intensity reflects the amplitude of the vibration of the vocal cord, and the duration is the time of vocal fold vibration. The timbre is determined by the vocal cords, the articulation method and the shape of the resonator. Singing is a special form of language expression. It can not only transmit information, but also convey emotion. It is an artistic expression form expressing emotion through sound. The pronunciation of the singing process is different from that of daily life, and there is also a difference in pitch, duration and intensity and timbre. In speech acoustic parameters, formant determines the vowel timbre. Formant is the area where the energy is relatively concentrated in the sound spectrum. During the process of pronounce or singing, when the sound passes through the resonator, it is affected by the filtering of the resonator, so that the sound energy is redistributed in different frequency domains. The frequency is enhanced by the resonance cavity, and the concentrated area in the time domain is called the formant. In the study of singing acoustics, mainly to study of acoustic properties of singer voice in the process of singing, which singer formant is an important indicator of singing acoustics.

This paper through the research methods of experimental phonetics. By collecting the singing voice signal from the traditional folk songs of the eastern part of Yugur, and analyzes the signal and acoustics parameters. So as to get the characteristics of the singing voice timbre of the folk songs in the eastern part of Yugur. 


\section{Experimental Design}

Signal Acquisition and Analysis. The signal acquisition environment is a professional audio recording studio. The indoor sound insulation effect is good, and has good sound-absorbing effect, low reverberation, so as to ensure the signal acquisition effect. Recording microphone is SONY clip-type microphone, with higher sensitivity. The sound card uses an external sound card, and a mixer using the Behringer 502 console. The acquisition device uses the Powerlab 16 channel collector. The analysis software uses PRAAT software to extract the required formant parameters.

The first is preparation, including consulting relevant reference materials. Followed by the signal acquisition, recording sampling frequency is $44100 \mathrm{~Hz}$, the resolution is 16 bits, save the format for the Windows PCM (*.wav) format. Finally, speech segmentation and data analysis, using Audition1.5 software for segmentation of the audio file, each word will save for .Wav format audio files, so as to facilitate the analysis of the latter processing. Extract the file parameters using the PRAAT software, and save the parameters into the Microsoft Excel table. The statistics of the acoustic parameters in Excel and display the statistical parameters in the form of charts.

Pronouncing People and Pronouncing Text. In this paper, The signal acquisition object for the Yugur folk songs heritage An Meiying. She at the age of seven with her family to learn folk songs. Her singing retains the singing methods and singing skills of the Yugur traditional folk songs. The voice of the inheritor is in good condition, no symptoms during acquisition, and the pronunciation is in good condition.

Collection of songs for eastern Yugur folk song on behalf of the repertoire of "Bay Horse". The song has melodious melody, and using the traditional folk songs to express the yearning for hometown and relatives.

\section{Experimental Results Analysis}

Characteristics Analysis of Singing Formant. The pronunciation of singing is different from that of the normal voice. Singing acoustics is the subject of studying the singing speech, which helps to measure the singers' pronunciation accurately. In the formant analysis, the singer formant is the main part of the study. The singer formant can enhance the penetrating power and sound intensity of the voice, which is usually possessed by well-trained singers. The singer formant was discovered by Bartholomew in the study of soprano singing in 1930s. According to the additional formant in the vocal spectrum, the concept of singer formant was proposed[1]. Later, Sundberg, an expert in Swedish acoustics, explains the cause of the singing formant in the study of singing formant[2]. Although there are still differences in the distribution range of singer formant, most of them think that the singer's formant male voice is around $2800 \mathrm{~Hz}$, and the female is around $3200 \mathrm{~Hz}$, which is roughly in the range of $2500 \mathrm{~Hz}-3500 \mathrm{~Hz}[3]$.

In this paper, the experiment object is female, so the location of the singer's formant should be at about $3500 \mathrm{~Hz}$. In the course of the study, in order to eliminate the influence of F1 and F2, as the energy distribution is easy to observe the singer's formant, use PRAAT software to extract the pronunciation of long vowels [a] and [i] formant data and the speech signals.

In the parameter extraction process, because the singer is female, so will the formant is set to the maximum of $5500 \mathrm{~Hz}$, and extracted 5 formants by using the number of normal speech formant measurements. In folk songs, select the longer part of the vowel stability section, excluding the impact of coarticulation of consonants and other vowels. The broadband spectrogram drawn by PRAAT is shown below. The abscissa is time, within 3 seconds, and the ordinate is the formant frequency. 


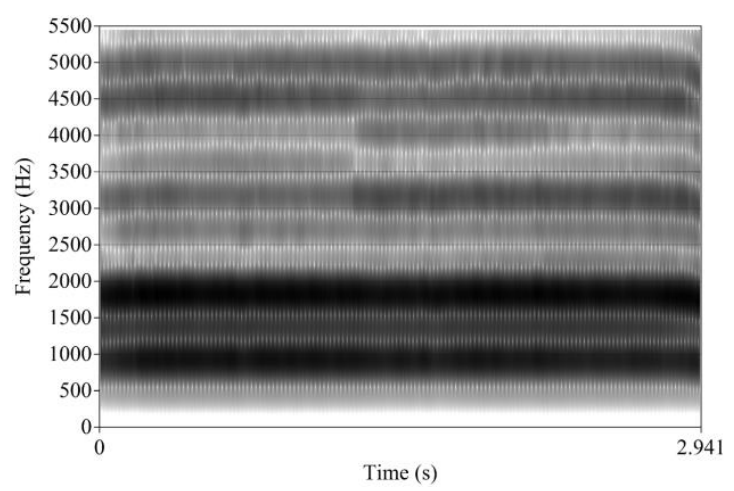

Figure 1. Broadband spectrogram for singing vowels [a]

As can be seen from the diagram, the vowel [a]'s F1 is around $800 \mathrm{~Hz}$, and the $\mathrm{F} 2$ is around $1700 \mathrm{~Hz}$. The frequency of the vowel F1 is inversely proportional to the level of the tongue, the lower the tongue, the greater the opening, the higher the formant frequency. F2 frequency is directly proportional to the tongue position, and the higher the F2 frequency before the tongue position is. The frequency of the F1 in the figure is approximately the same as that of the normal speech, while the frequency of the F2 is higher than the formant frequency of the normal speech, and the F2 of the normal speech is about $1200 \mathrm{~Hz}$. As can be seen in the diagram, there is a more concentrated part of the energy in the range of $3000 \mathrm{~Hz}-3500 \mathrm{~Hz}$, and there is also an obvious energy concentration zone in the high frequency energy region, roughly within the range of $4500 \mathrm{~Hz}-5000 \mathrm{~Hz}$. Therefore, it is possible to determine the singer formant appeared in the $3000 \mathrm{~Hz}-3500 \mathrm{~Hz}$ range.

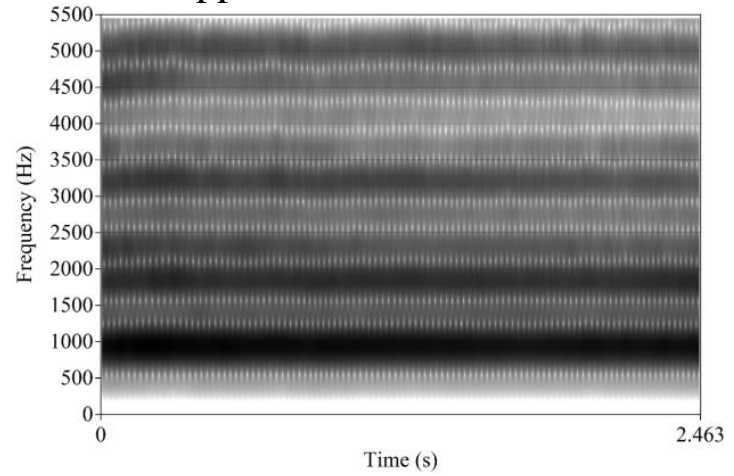

Figure 2. Broadband spectrogram for singing vowels [i]

In the singing vowel [i], the frequency of $\mathrm{F} 1$ is about $700 \mathrm{~Hz}$, and the frequency of $\mathrm{F} 2$ is near $2000 \mathrm{~Hz}$. The frequency of F1 and F2 is approximately the same as the resonant frequency of normal speech, but in the high frequency part in the range of $3000 \mathrm{~Hz}-3500 \mathrm{~Hz}$ the singer's formant, and the range of $4500 \mathrm{~Hz}-5000 \mathrm{~Hz}$ has the strong energy concentration area. The distribution of the high frequency formant is similar to that of vowel [a] in the high frequency part. Because singing belongs to the original ecological singing, without the influence of accompaniment, formant reflects the singing of the original appearance. Therefore, in the eastern Yugur folk songs, female singer resonance peaks in the range of $3000 \mathrm{~Hz}-3500 \mathrm{~Hz}$, and the distribution of energy concentrated in the high frequency position. This shows that the original singer also mastered the singing skills, with a standard singer formant performance. 


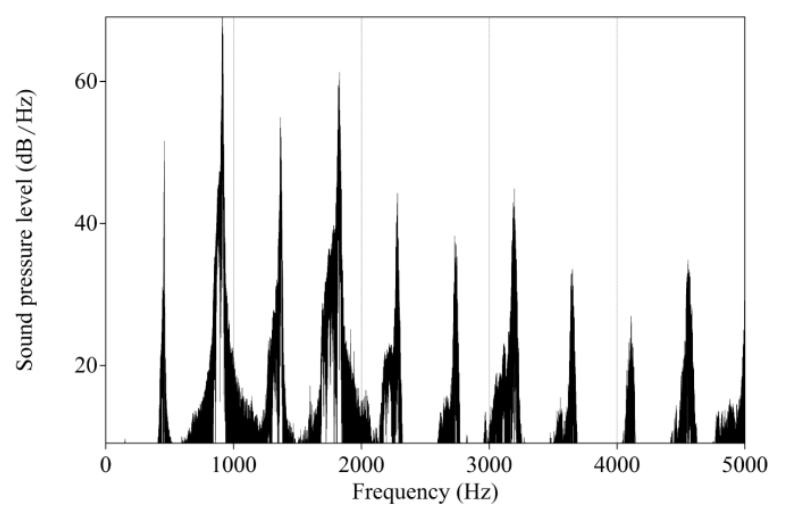

Figure 3. The long time average power spectrum of vowel

In order to further study the singer's formant. Eliminate the consonants, the shorter vowel parts, and the blank speech section of the whole song, which retain only the vowel part of the speech. And then extract the long-term average power spectrum according to the singing vowel. In the figure, the abscissa is the frequency and the ordinate is the sound pressure level. In the $1000 \mathrm{~Hz}$ and $2000 \mathrm{~Hz}$ as the center of the two energy concentration areas, corresponding to F1 and F2. And in the $3000 \mathrm{~Hz}-3500 \mathrm{~Hz}$ frequency range also appeared higher energy distribution, corresponding to the singer's formant location. Therefore, it shows that the original singer has better singing skills and master singing skills.

Singing Voice Frequency Domain Characteristic Analysis. Singing voice is the same as that of normal communicative voice, which is produced by human vocal organs and modulated by the resonant cavity. Especially in the singing process, it is necessary to adjust the pitch, duration and timbre of voice while singing, among them, resonance plays an important role. There is a rich variety of expressiveness. Compared with the normal speech voice, Compared with the normal speech voice, the number of resonator cavity and the influence of the resonance method, the intensity of the singing voice is higher than the voice of speech. In the singing voice there is also the pitch and the harmonic. Influenced by the frequency of the vocal cords, the vocal frequencies of the singing are higher than the frequency of speech. The vocal cords are tenser when they are singing, but the vocal cords are less tense when talking[4]. The emergence of singer formant also shows that people have more high frequency resonance components to participate in singing and play an important role(Wu jing2007).

The tone is determined by the pitch frequency. Frequency domain of pitch is in the process of the pronunciation of tones from low to high, mainly affected by the regulation effect of vibration of the vocal cords and resonance cavity. The vibration of the vocal cords in singing will affect the vocal range of the singer. In the process of pitch extraction, the pitch frequency value of the whole song was extracted, and the consonants were removed. Parameters are calculated by the SPSS19.0 software.

Table 1 Pitch frequency $(\mathrm{Hz})$

\begin{tabular}{|l|l|r|}
\hline $\mathrm{N}$ & Valid & 10687 \\
\cline { 2 - 3 } & Missing & 0 \\
\hline Mean & 342.60 \\
\hline Median & 342.09 \\
\hline Std. Deviation & 85.12 \\
\hline Range & 432.10 \\
\hline Minimum & 166.75 \\
\hline Maximum & 598.85 \\
\hline
\end{tabular}

Usually, people in the process of communication, the pitch frequency range between $150-320 \mathrm{~Hz}$, 
the male lowest is about $80 \mathrm{~Hz}$ and the female highest can reach about $400 \mathrm{~Hz}$, the pitch range of singing can reflect the singer's singing ability. In the course of the study, the statistical results of the pitch frequency parameters are shown in Figure 1. In the pitch frequency table, 10687 fundamental values of the whole folk song are selected. The singer has an average pitch frequency of $342.6 \mathrm{~Hz}$, a maximum of $598.85 \mathrm{~Hz}$, and a minimum of $166.75 \mathrm{~Hz}$. The resulting pitch frequency range is 432.1Hz.

In the process of studying the singing voice (Wu jing2007), it is concluded that the untrained singer is a half octave range, trained voice range for two and a half or two octaves, rare singer can reach three octaves. From the data in the table, it can be concluded that the singer's singing voice is lower than bel canto, such as the alto range between 160-640Hz. Therefore, in the original ecological singing, because of the degree of singing skill and training, the base frequency is generally low. From the singing pitch range, pitch range of the eastern Yugur folk singer is also wider, can reach more than $400 \mathrm{~Hz}$.

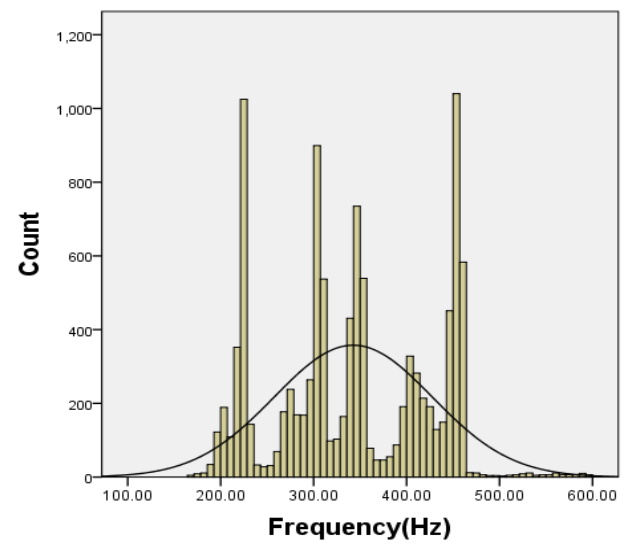

Figure 4. Pitch frequency histogram

Pitch frequency is one of the important parameters of singing artistic voice, and it has an important influence on the timbre of singing. The fundamental difference between normal speaking voice and singing voice is the vocal cord is a continuous vibration during the singing process. However, in the process of pronunciation, voiceless vocal cords do not vibrate. The singing pitch frequency reflects the range of the singer's pitch from low to high when singing.

In order to analyze the distribution of the singing pitch frequency, we can further observe it through the histogram. In the histogram of figure 4, there are four peaks, and the whole parameter is presented in a normal distribution. The pitch frequency of four peak are the first $235 \mathrm{~Hz}$, second $305 \mathrm{~Hz}$, third $350 \mathrm{~Hz}$, fourth $465 \mathrm{hz}$, of which the first frequency and the fourth part of the emergence of the highest frequency. It shows that during the singing process, the singer's pitch frequency was concentrated at about $235 \mathrm{~Hz}$, and the pitch frequency was concentrated at about $465 \mathrm{~Hz}$. From the distribution characteristics, not only reflects the changes in the level of singing tunes, the more high-frequency distribution, but also shows that the sound of the song is bright. In the whole distribution, the highest frequency of the pitch frequency is the high frequency part, which shows that the singer sings more with real voice when singing. After the adjustment of resonance, the sound is bright and loud, which is in line with the characteristics of the original folk songs[5].

\section{Conclusion and Discussion}

The eastern folk song is one of the Chinese minority folk songs, folk songs of the comprehensive characteristics of Mongolia long harmonic and Yugur folk songs. The original singing style, no musical accompaniment, and most of singers for oral way acquisition of folk songs. This article through singing voice signal acquisition of Eastern Yugur folk songs, through the acoustic analysis, obtains the folk song singing timbre and singer's singing acoustic characteristic[6].

Acoustic properties of acoustic by studying singing songs. The study found that the Yugur singer through singing learning, the singer's formant is produced in the range of $3000 \mathrm{~Hz}-3500 \mathrm{~Hz}$. It shows 
that the Yugur original singing while there is no system theory in the process of singing, but the singer has the singing method and the acquisition rule, and has the singing skill, also has the good singing level. In the process of singing, the airflow impacts the vocal cords, causes the vocal cords to vibrate, and the glottal signals result in the adjustment of the resonant cavity, resulting in the beautiful and sonorous singing that people can hear. Part in the process of singing, singing is focused on the vowel. Most pitch frequency distribution in the high frequency region. It shows that the vocal cords vibrate faster in the singing process, and the singers mostly sing with the true voice. It reflects the bright features of the eastern Yugur folk songs.

In the future research, we will make a comprehensive study of singers of different degrees. Therefore, it is a good reference to provide acoustics for folk song learners, and play a role in the protection and inheritance of folk songs.

\section{Acknowledgement}

This research was financially supported by Northwest MinZu University 2016 annual basic scientific research funds of the central university funding projects (Grant NO. 31920160072).

\section{Reference}

[1].Sundberg J. The acoustics of the singing voice[J]. Scientific American, 1977.

[2]. Oncley P B. Acoustics of the Singing Voice[J]. Journal of the Acoustical Society of America, 1954.

[3].Howard D M. Acoustics of the trained versus untrained singing voice[J]. Current Opinion in Otolaryngology \& Head \& Neck Surgery, 2009.

[4].Wu Jing. An Acoustics Explanation to the Timbre of the Chinese Traditional Singing-Taking Female Voiceasan Example, Post graduate Program, China Art Aeademy 2007.

[5].Du Yaxiong. Introduction of Yugur folk songs . people's music, 1981.

[6].Wang Shiqian. The origin and some discussions on the concept of singer formant. . applied acoustics, 1987. 